\title{
Sub-wavelength resonances in polygonal metamaterial cylinders
}

\author{
Arslanagic, Samel; Breinbjerg, Olav
}

Published in:

IEEE APS Int. Symp. /USNC/URSI Nat. Radio Science Meeting

Link to article, DOI:

10.1109/APS.2008.4619992

Publication date:

2008

Document Version

Publisher's PDF, also known as Version of record

Link back to DTU Orbit

Citation (APA):

Arslanagic, S., \& Breinbjerg, O. (2008). Sub-wavelength resonances in polygonal metamaterial cylinders. In IEEE APS Int. Symp. /USNC/URSI Nat. Radio Science Meeting (pp. 1-4). IEEE.

https://doi.org/10.1109/APS.2008.4619992

\section{General rights}

Copyright and moral rights for the publications made accessible in the public portal are retained by the authors and/or other copyright owners and it is a condition of accessing publications that users recognise and abide by the legal requirements associated with these rights.

- Users may download and print one copy of any publication from the public portal for the purpose of private study or research.

- You may not further distribute the material or use it for any profit-making activity or commercial gain

- You may freely distribute the URL identifying the publication in the public portal

If you believe that this document breaches copyright please contact us providing details, and we will remove access to the work immediately and investigate your claim. 


\title{
Sub-Wavelength Resonances in Polygonal Metamaterial Cylinders
}

\author{
Samel Arslanagic and Olav Breinbjerg*, \\ DTU Elektro, ElectroScience Section, Technical University of Denmark, \\ Building 348, Ørsteds Plads, DK-2800 Kgs. Lyngby, Denmark, \\ Tel: +45 4525 3800, Fax: +45 4593 1634; E-mail: sar@oersted.dtu.dk
}

\section{Introduction}

Recent metamaterial (MTM) research has demonstrated the potential of double- and single-negative (DNG and SNG) materials, as well as combinations of these with doublepositive (DPS) materials, for sub-wavelength waveguides, cavities, scatterers, and radiators of different canonical shapes [1]-[5]. For the cylindrical geometry it was shown in [3] that a set of concentric circular MTM cylinders excited by a near-by electric line current possesses sub-wavelength resonances where the excitation of specific modes leads to large radiated power for constant line current.

This work investigates how these resonances are affected by the shape of the cylinder; in particular, its deviation from the perfect circular shape. To this end a set of concentric polygonal cylinders excited by an electric line current is analyzed numerically and it is shown that these structures also possess sub-wavelength resonances - similar to those of the circular cylinders. The analysis includes the near-field distribution as well as the radiation resistance in the case of simple, but lossy and dispersive, MTMs. The time factor $\exp (j \omega t)$, with $\omega$ being angular frequency and $t$ time, is assumed and suppressed.

\section{Theory}

The circular and polygonal configurations are depicted in Figure 1.
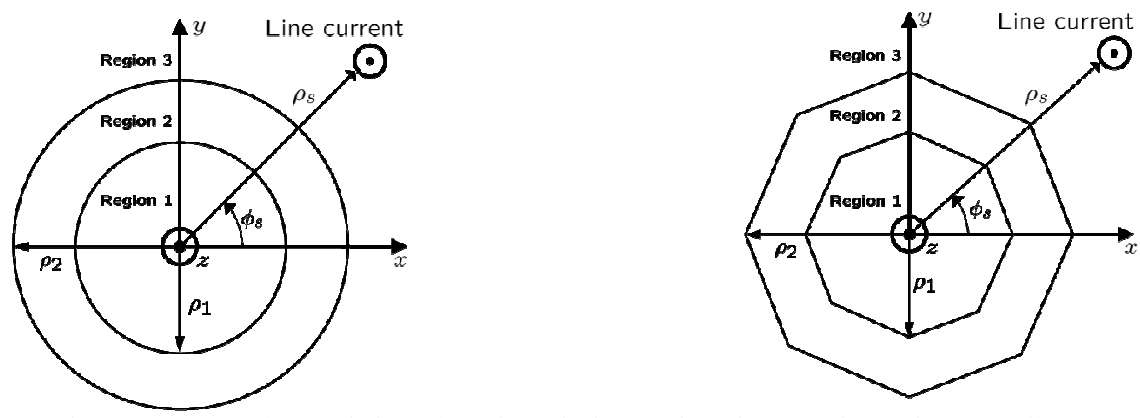

Figure 1. The cross-section of the circular (left) and polygonal (right) configurations.

To the left, a circular cylinder (region 1) of radius $\rho_{1}$ is covered by a circular shell (region 2) of outer radius $\rho_{2}$. To the right, a regular polygonal cylinder (region 1), with a circumscribed circle of radius $\rho_{1}$, is covered by a regular polygonal shell (region 2) with a circumscribed circle of radius $\rho_{2}$. For both configurations, regions 1 and 2 are surrounded by free space (region 3 ) with the permittivity $\varepsilon_{0}$ and the permeability $\mu_{0}$. Moreover, regions 1 and 2 are composed of simple, lossy, and dispersive DPS, DNG, or SNG materials with a permittivity, $\varepsilon_{i}=\varepsilon_{i}^{\prime}-j \varepsilon_{i}^{\prime \prime}$, and a permeability, $\mu_{i}=\mu_{i}^{\prime}-j \mu_{i}^{\prime \prime}$, $(i=1(2)$ for region $1(2))$. The cylinders are illuminated by an infinite electric line 
current $I_{e}$ that is parallel to the cylinders and can be located in any of the three regions. The cylindrical $(\rho, \varphi, z)$-coordinate system and the Cartesian $(x, y, z)$-coordinate system are introduced with the $z$-axis coinciding with the common axis of the cylinders. The coordinates of the line current are $\left(\rho_{s}, \varphi_{s}\right)$.

For the circular cylinder configuration an exact solution has been established using the eigenfunction expansion technique. The known line current field, as well as the unknown fields in the three regions, are expanded in terms of cylindrical wave functions, and the unknown expansion coefficients are determined by enforcing the boundary conditions at the interfaces between the three regions; please see [3] for details.

For the polygonal cylinder configuration, a numerical solution has been established using the HFSS software [6]. To this end, finite length MTM cylinders and a finite length current tube, of radius $a$, current $I_{e}$, and centered at $\left(\rho_{s}, \varphi_{s}\right)$, is positioned between, and perpendicular to, 2 parallel, perfectly conducting, square plates with side length $w$ and separation $h$. Between the edges of these plates, uniform perfect matching layers of thickness $d$, and joint corners and edges, are inserted. The parameters $a=$ $0.15 \mathrm{~mm}, h=1 \mathrm{~mm}, w=600 \mathrm{~mm}$, and $d=12.28 \mathrm{~mm}$.

\section{Results and Discussion}

According to [3] a dipole mode resonance occurs for the circular cylinder configuration if region 1 is free space, region 2 is a SNG material with $\left(\varepsilon_{2}, \mu_{2}\right)=\left(1 \varepsilon_{0},-4 \mu_{0}\right)$, and $\left(\rho_{1}, \rho_{2}\right)=(6,10.0329) \mathrm{mm}$. It is now investigated if a similar resonance occurs for the polygonal cylinder configuration. Both configurations have the same material parameters, radius $\rho_{1}$, current $I_{e}=1 \mathrm{~A}$, and frequency of operation $f_{0}=300 \mathrm{MHz}$.

Figure 2(left) shows the radiation resistance $R_{\text {rad }}$ as a function of the outer radius $\rho_{2}$ for the $n$-sided polygonal cylinders with $n=48, n=24, n=12$ and $n=8$, for $\left(\rho_{s}, \varphi_{s}\right)=\left(5.75 \mathrm{~mm}, 0^{\circ}\right)$. The results for the circular cylinder configuration are also included. It is observed that the resonance occurs also for the polygonal cylinder configurations but at a slightly different value of $\rho_{2}$ depending on the number of sides $n$. For large $n$ the outer radius $\rho_{2}$ at resonance, and the radiation resistance $R_{\text {rad }}$, is close to that of the circular cylinder configuration. As $n$ decreases, and the polygonal cylinder thus deviates considerably from the circular cylinder, the outer radius $\rho_{2}$ increases slightly and the radiation resistance $R_{\text {rad }}$ more notably. These values are summarized in Table 1. For comparison, the line current in free space has $R_{\text {rad }}=0.592 \Omega / \mathrm{mm}$.
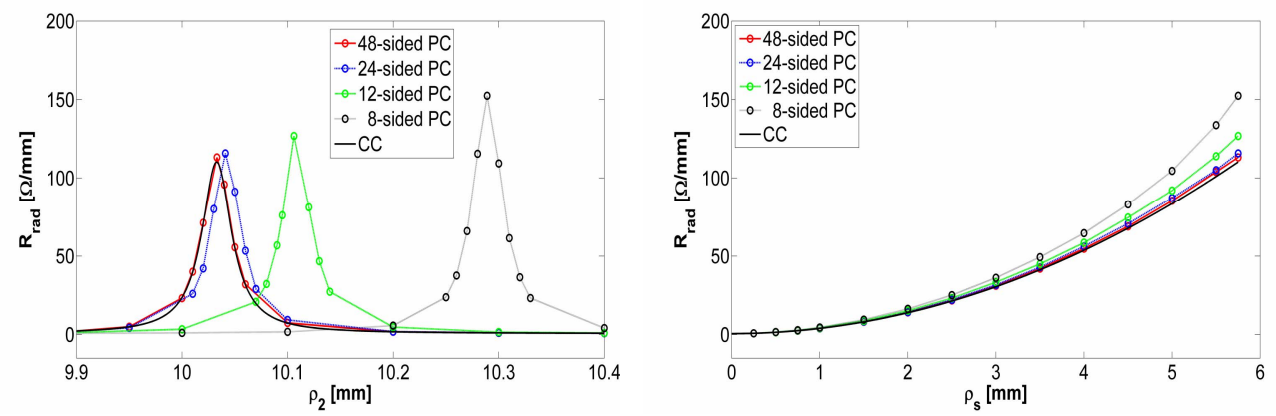

Figure 2. Radiation resistance as a function of outer radius $\rho_{2}$ (left) and line/tube current position $\rho_{s}$ (right) for the circular (CC) and polygonal (PC) configurations. 
Figure 2 (right) shows $R_{\text {rad }}$ as a function of the location of the line current, $\rho_{s}$, in region 1 for the different resonant configurations. Since the difference between $R_{\text {rad }}$ for the circular

\begin{tabular}{|c|c|c|c|c|c|}
\hline Quantity & Circular & $n=48$ & $n=24$ & $n=12$ & $n=8$ \\
\hline $2 \quad[\mathrm{~mm}]$ & 10.0329 & 10.033 & 10.041 & 10.106 & 10.289 \\
\hline$R_{\text {rad }}[\Omega / \mathrm{mm}]$ & 110.2 & 113.2 & 115.8 & 126.8 & 152.4 \\
\hline
\end{tabular}

and polygonal cylinders is profound only for $\rho_{s}$ close to

Table 1. Important parameters at the resonance. $\rho_{1}$, it follows that the shape of the cylindrical structure is not of primary importance and that the results for the circular cylinders can also be obtained with polygonal cylinders.

In Figure 3, the magnitude of the electric field is shown at positions in the $x y$ plane for the circular cylinders as well as the 24-, 12-, and 8-sided polygonal cylinders. In all cases, a dipolar mode is observed which implies that the resonances in Figures 1 and 2 are indeed due to the excitation of this mode. It is noted that the fields of the polygonal cylinders attain higher values and become more confined near the corners as the number of sides decreases.
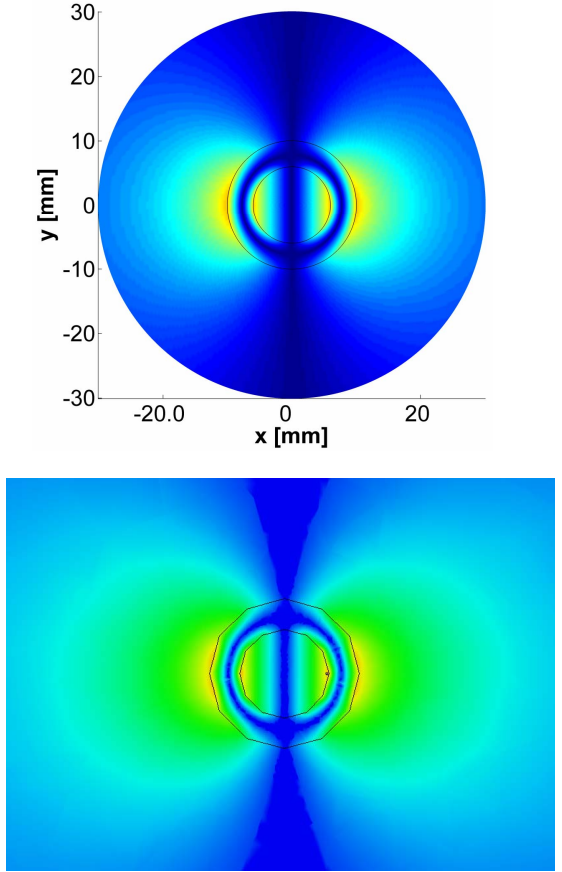
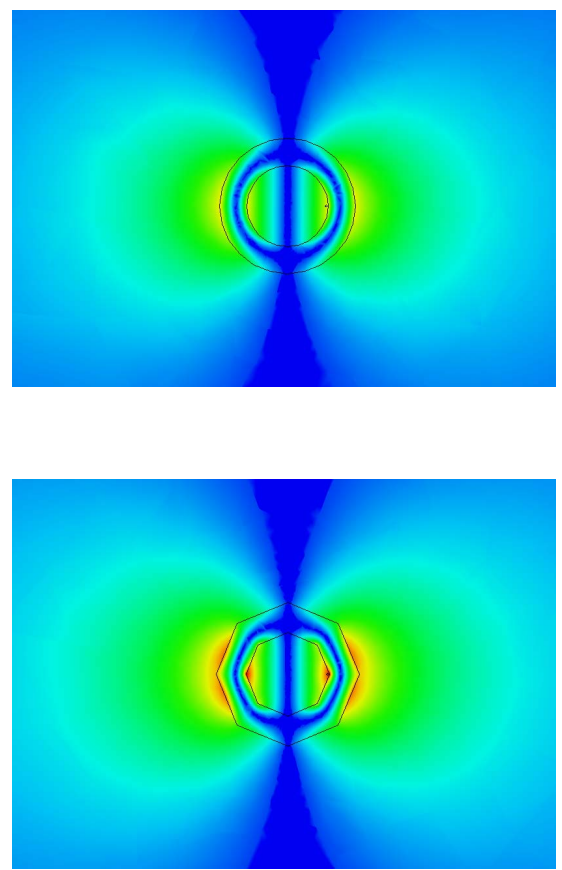

Figure 3. The electric field for the resonant circular (top left) and for the 24-side (top right), 12-side (bottom left), and 8-side (bottom right) polygonal configurations. For all 4 plots, the linear dynamic range is set to [150-170000] V/m.

To asses the effects of loss and dispersion in the MTM, the lossy Drude and Lorentz models [3] have been introduced and this leads to the results in Figure 4. Clearly, the resonances occur in all cases. While they are broad-band in the non-dispersive case with high values of $R_{\text {rad }}$, they narrow considerably when dispersion is present and the value of $R_{\text {rad }}$ decreases significantly; this is particularly true for the Lorentz model. However, it is seen that the results for the polygonal cylinders are close to those of the circular cylinders. This implies that it is the dispersion model rather than the geometrical shape that determines the behavior of the resonances. 

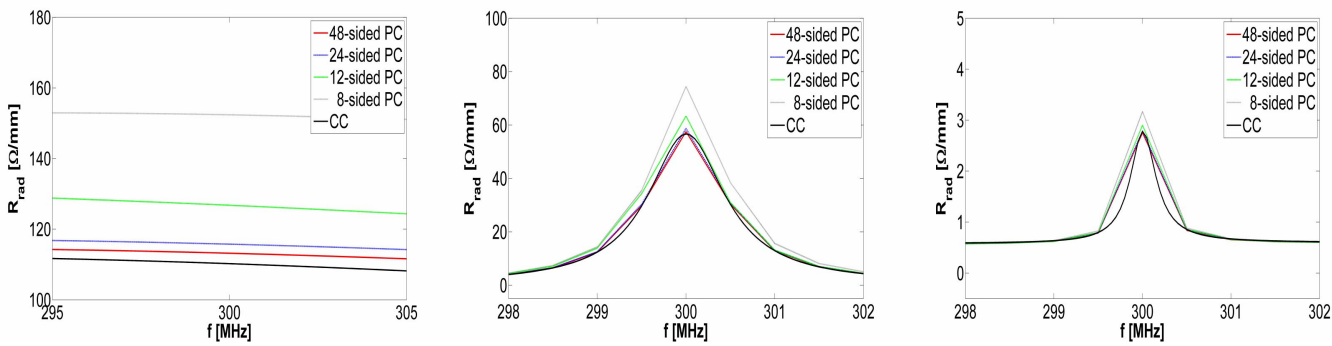

Figure 4. Radiation resistance as a function of frequency for the resonant cylindrical (CC) and polygonal (PC) configurations with no dispersion (left), Drude dispersion (middle), and Lorentz dispersion (right).

Finally, it is important to note that the resonances of the polygonal cylinders are not restricted to locations of the line current near corners. Figure 5 shows that a dipole mode occurs also for the line current near the middle of a face at $\left(\rho_{s}, \varphi_{s}\right)=\left(5.29 \mathrm{~mm}, 22.5^{\circ}\right)$.

Figure 5. The electric field of the resonant 8sided polygonal cylinders with the line current near the side of the cylinders. The linear dynamic range is set to [150-170000] $\mathrm{V} / \mathrm{m}$.

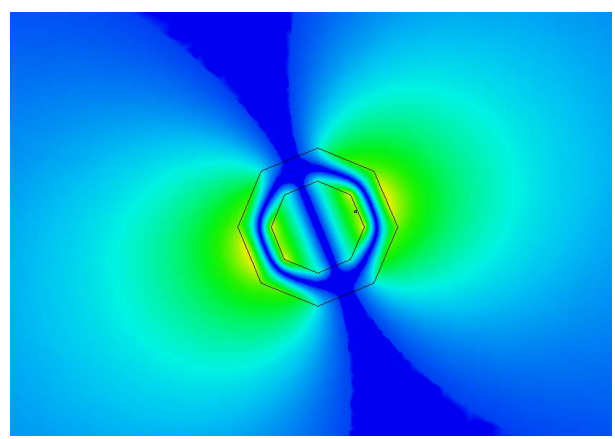

\section{Conclusions}

It has been shown that the sub-wavelength resonances of circular MTM cylinders also occur for polygonal MTM cylinders. This is the case for lossless and non-dispersive cylinders as well as lossy and dispersive cylinders. The sub-wavelength resonances are thus not limited to structures of canonical shapes but occurs also for other shapes and they are determined more by the material parameters than the geometrical parameters.

\section{Acknowledgments}

This work is supported by the Danish Research Council for Technology and Production Sciences within the TopAnt project. Aycan Erentok is acknowledged for assisting with HFSS calculations.

\section{References}

[1] N. Engheta and R. W. Ziolkowski, "A Positive Future for Double Negative Metamaterials," IEEE Microwave Theory Tech., vol. 53, pp. 1535-1556, Apr. 2005.

[2] A. Alú, and N. Engheta, "Resonances in sub-wavelength cylindrical structures made of pairs of double-negative and double-positive or epsilon-negative and mu-negative coaxial shells," ICEAA, Turin, Italy, Sep. 8-12, 2003.

[3] S. Arslanagic, R. W. Ziolkowski and O. Breinbjerg, "Analytical and numerical investigation of the radiation and scattering from concentric metamaterial cylinders excited by an electric line source," Radio Sci., vol. 42, RS6S15, Nov. 2007.

[4] S. Arslanagic, R. W. Ziolkowski and O. Breinbjerg, "Analytical and numerical investigation of the radiation from concentric metamaterial spheres excited by an electric Hertzian dipole," Radio Sci., Vol. 42, RS6S16, Oct. 2007.

[5] H. Wallén, H. Kettunen, and A. Sihvola, "Electrostatic resonances of negativepermittivity interfaces, spheres and wedges,"The First Intl. Congress on Advanced Electromagnetic Materials for Microwave and Optics, Rome, Italy, Oct. 22-26, 2007.

[6] ANSOFT HFSS, Version 10.1.3, Copyright (C) 1984-2006 Ansoft Corporation. 